\title{
Semisimple rings of quotients
}

\section{Julius M. Zelmanowitz}

\begin{abstract}
Necessary and sufficient conditions on an arbitrary Gabriel filter of left ideals of a ring $R$ are determined in order that the ring of quotients of $R$ with respect to the filter be semisimple artinian. Special instances include generalizations of earlier work on classical rings of quotients and maximal rings of quotients.
\end{abstract}

\section{Introduction}

There have been several interesting results which determine when a ring of quotients of a ring is semisimple artinian. For the classical ring of quotients $Q$ with respect to the set of regular elements of a ring $R$, Levy proved in [5] that $Q$ is semisimple artinian if and only if torsionfree divisible $R$-modules are injective. In [7], Sandomierski characterized rings with a semisimple artinian maximal quotient ring as being nonsingular and finite dimensional. More generally, for the ring of quotients with respect to a Gabriel topology, some progress has been made in [1] and [6] for the case of torsion-free rings. Hereditary rings with semisimple artinian rings of quotients are studied in [3].

The purpose of this article is the determination of when the ring of quotients with respect to an arbitrary Gabriel topology is semisimple artinian. This is accomplished in $\$ 2$. In $\$ 3$ and $\$ 4$, respectively, the above-cited results of Sandomierski and Levy are shown to be special cases of the main theorem, and are extended to allow for the possibility of torsion. In $\$ 5$, injective ideals of a ring of quotients are examined; this permits one to learn when the ring of quotients is simple. Some related observations are presented in $\$ 6$.

Received 3 July 1978. 
1 .

Since the notation and language of torsion theories are not quite standardized, we will first indicate the usages of this article. Two basic references for the rudiments of the subject are [2] and [8].

By a module we mean a left $R$-module over some ring $R$. Actually, in all that follows $R$ need not have an identity element, provided that one insists that all modules on wich $R$ acts trivially are torsion, and that one makes slight modifications in definitions and statements of theorems (such as replacing $R$ by $R^{l}$ ). For simplicity however, we assume that $R$ contains an identity element. For subsets $N$ and $N^{\prime}$ of a module $M$, we set $\left(N: N^{\prime}\right)=\left\{r \in R \mid r N^{\prime} \subseteq N\right\}$.

Throughout this paper $F$ will denote a Gabriel topology of left ideals of $R$ with $O F F$. That is, $F$ satisfies

$$
\begin{aligned}
& \text { (i) } I \in F \text { and } a \in R \text { implies }(I: a) \in F \text {, and } \\
& \text { (ii) }(J: a) \in F \text { for all } a \in I \in F \text { implies that } J \in F \text {. }
\end{aligned}
$$

Such an $F$ is necessarily a filter. We let

$$
T(M)=T_{F}(M)=\{m \in M \mid(0: m) \in F\},
$$

the F-torsion submodule of a module $M$. A module is F-torsion if $T_{F}(M)=M$, and F-torsion-free if $T_{F}(M)=0$. A submodule $N$ of $M$ is F-dense in $M$ if $M / N$ is F-torsion, and $N$ is F-closed in $M$ if $M / N$ is F-torsion-free. We also set $N^{C}$ equal to the submodule defined by $N^{C} / N=T(M / N) ; N^{C}$ is an F-closed submodule of $M$, called the F-closure of $N$. The class of F-torsion modules is closed under submodules, homomorphism, extension and direct sums; while the class of F-torsion-free modules is closed under isomorphism, submodules, extension, injective hulls and direct products. When no confusion can arise, we will delete the prefix "F-" from the preceding terms, and speak simply of torsion modules, dense submodules, and so on.

A module $M$ is F-injective if every $f \in \operatorname{hom}_{R}(I, M)$ with $I \in F$ can be extended to an element of $\operatorname{hom}_{R}(R, M) ;$ equivalently, $T(\hat{M} / M)=0$ where $\hat{M}$ denotes the $R$-injective hull of $M$. Every module has an 
F-injective hull $F(M)$ obtained as $F(M) / M=T(\hat{M} / M)$; note that $M$ is dense in $F(M)$, and $F(M)$ is closed in $\hat{M}$. One defines $M_{F}$, the module of quotients of $M$ with respect to $F$, as $F(M / T(M)) ; M_{F}$ is torsionfree and F-injective. $R_{F}=F(R / T(R))$ forms a ring called the ring of quotients of $R$ with respect to $F$; and the multiplication on $R_{F}$ extends its $R$-module structure. The assignment $M \rightarrow M_{F}$ yields a left exact functor from $R$-modules to $R_{F}$-modules.

As it is our intention to make this article reasonably self-contained, we now list some elementary and well-known observations that are required in the sequel.

(1.1). If $N_{1} \subseteq N_{2} \subseteq N_{3}$ is a trio of submoduzes and $N_{i}$ is dense (respectively, closed) in $N_{i+1}$ for $i=1,2$, then $N_{1}$ is dense (respectively, closed) in $N_{3}$.

This is because the torsion (respectively, torsion-free) modules are closed under extension. $1 /$

(1.2). A dense submoduze $N$ of a torsion-free module $M$ is essential in $M$.

For given $0 \neq m \in M, \quad(N: m) \in F$ and $0 \neq(N: m) m \subseteq R m \cap N$. //

(1.3). If $I$ is a left ideal of $R$ and $I+T(R) \in F$ then $I \in F$.

To see this note that $I$ is dense in $I+T(R)$ since

$$
I+T(R) / I \cong T(R) / I \cap T(R)
$$

and then apply (1.1). //

(1.4). A closed submodule $N$ of an F-injective module $M$ is F-injective.

$M=F(M)$ is closed in $\hat{M}$, so $N$ is closed in $\hat{M}$ by (1.1). Hence $N$ is closed in $\hat{N} \subseteq \hat{M}$; that is, $N=F(N)$. $/ /$

LEMMA 1.5. Set $\bar{F}=\{\bar{I} \mid I \in F\}$, a family of left ideals of $\bar{R}=R / T_{F}(R)$. Then

(i) $\bar{I} \in \bar{F}$ if and only if $\bar{I}$ is $F$-dense in $\bar{R}$; 
(ii) $\bar{F}$ is an idempotent filter of left ideals of $\bar{R}$;

(iii) $T_{\bar{F}}(\bar{R})=\overline{0}$;

(iv) $R_{F}=\bar{R}_{\bar{F}}$.

Proof. (i) follows from (1.3) and is in fact true for any factor ring of $R$.

(ii) is also true for every factor ring of $R$. The proof is routine.

(iii) If $\bar{a} \in T_{\bar{F}}(\bar{R})$, then $\left(T_{F}(R): a\right) \in F$. But $T_{F}(R)$ is F-closed in $R$, so $\bar{a}=\overline{0}$.

(iv) We first note that $R_{F}$ is an $\bar{R}$-module, and that $R_{F}$ is F-injective. To see the latter assertion, let $\bar{I} \in \bar{F}$ and $f \in \operatorname{hom}_{\bar{R}}\left(\bar{I}, R_{F}\right)$ be given; we must extend $f$ to an element of hom $\bar{R}\left(\bar{R}, R_{F}\right)$. Let $\pi$ denote the canonical epimorphism of $R$ onto $\bar{R}$. Then $f \circ \pi \in \operatorname{hom}_{R}\left(I, R_{F}\right)$ with $I \in F$. Since $R_{F}$ is F-torsion-free and F-injective, $f \circ \pi$ has a unique extension $g^{\prime} \in \operatorname{hom}_{R}\left(R, R_{F}\right)$. Now ker $g^{\prime} \supseteq T_{F}(R)$, so $g^{\prime}$ induces a unique homomorphism $g \in \operatorname{hom}_{R}\left(R, R_{F}\right)$ with $g \circ \pi=g^{\prime}$. Thus $g$ is an $\bar{R}$-homomorphism and $\left.(g \circ \pi)\right|_{I}=\left.g^{\prime}\right|_{I}=f \circ \pi$, so $g$ extends $f$.

Since $T_{F}(\bar{R})=\overline{0}$, it remains only to prove that $\bar{R}$ is $\bar{F}$-dense in $R_{F}$. But this is evident, and we are done. //

2 .

We begin by presenting the main result, for which we require the following definition. A set $S$ of left ideals of $R$ will be called cofinally finite if given any $I \in S$ there exists a finitely generated left ideal $J \subseteq I$ with $J$-dense in $I$.

THEOREM 2.1. The following conditions are equivalent:

(1) $R_{F}$ is semisimple artinian;

(2) $R$ satisfies the ascending chain condition on closed left ideals, and torsion-free F-injective modules are injective; 
(3) F is cofinally finite, and torsion-free F-injective modules are injective.

Proof. (1) $\Rightarrow(2)$. The fact that $R$ satisfies the ascending chain condition on closed left ideals follows immediately from the observation that $\psi^{-1}\left(R_{F} \bar{I} \cap \bar{R}\right)=I$ for any closed left ideal $I$ of $R$, where $\psi$ denotes the canonical homomorphism of $R$ onto $\bar{R}=R / T(R)$. Indeed, for the same conclusion, it would clearly suffice to have $R_{F}$ noetherian (see also $[2, p .136])$. To see this formula, in turn, note that $I \subseteq \psi^{-1}\left(R F^{\bar{I}} \cap \bar{R}\right)$, and that for any $x \in \psi^{-1}\left(R_{F} \bar{I} \cap \bar{R}\right)$, $(I: x)=(\bar{I}: \bar{x}) \in F$. Since $I$ is closed we learn that $I=\psi^{-1}\left(R_{F} \bar{I} \cap \bar{R}\right)$.

Next, let $M$ be a torsion-free F-injective $R$-module. Then $M=M_{F}$ is an $R_{F}$-module, as is the $R$-injective hull $\hat{M}$ of $M$. Since all $R_{F}$-modules are injective, $M$ must be an $R_{F}$-direct summand of $\hat{M}$. But then necessarily $M=\hat{M}$.

$(2) \Rightarrow(3)$. We prove that the set of all left ideals of $R$ is cofinally finite. For let $I$ be any nonzero left ideal of $R$. Choose $0 \neq a_{1} \in I$. If $R a_{1}$ is dense in $I$, we are done. If not, $\left(R a_{1}\right)^{c} \pm I$, where $\left(R a_{1}\right)^{c}$ denotes the closure of $R a_{1}$ in $R$. Choose $0 \neq a_{2} \in I \backslash\left(R a_{1}\right)^{c}$; then $\left(R a_{1}\right)^{c} \varsubsetneqq\left(R a_{1}+R a_{2}\right)^{c}$. If $R a_{1}+R a_{2}$ is dense in $I$, we are done. If not, $\left(R a_{1}+R a_{2}\right)^{c} \notin I$, and we may continue this construction. Since $R$ satisfies the ascending chain condition on closed left ideals, the construction must terminate. Thus for some integer $n$ there exists $R a_{1}+\ldots+R a_{n}$ dense in $I$.

$(3) \Rightarrow(1)$. We first show that every finitely generated left ideal of $R_{F}$ is injective. For any $x \in R_{F}$, we have the exact sequence

$$
0 \rightarrow(0: x)_{R_{F}}>R_{F} \rightarrow R_{F} x \rightarrow 0
$$


where $(0: x)_{R_{F}}=\left\{r \in R_{F} \mid r x=0\right\}$. Now $R_{F}$ is F-injective, and $(0: x)_{R_{F}}$ is closed since $R_{F} x$ is torsion-free, so by $(1.4), \quad(0: x)_{R_{F}}$ is F-injective. By our hypothesis then, $(0: x)_{R_{F}}$ is injective. It follows that $R_{F} x$ is injective for any $x \in R_{F}$.

Next, consider the exact sequence

$$
0 \rightarrow \operatorname{ker} \Sigma \rightarrow \underbrace{t}_{i=1} R_{F} x_{i} \stackrel{\sum}{\longrightarrow} \sum_{i=1}^{t} R_{F} x_{i} \rightarrow 0
$$

where $\Sigma$ is the canonical epimorphism. Then as in the previous paragraph, $\operatorname{ker} \Sigma$ is closed. Since $\bigoplus_{i=1}^{t} R_{F} x_{i}$ is injective, it follows that ker $\Sigma$ is torsion-free and F-injective, hence injective. Thus the sequence splits, and $\sum_{i=1}^{t} R_{F} x_{i}$ is injective.

Now let $I \in F$ be given. Since $F$ is cofinally finite, there exists $\sum_{i=1}^{t} R a_{i} \in F$ with $\sum_{i=1}^{t} R a_{i} \subseteq I$. Then $\sum_{i=1}^{t} R_{F} \bar{a}_{i} \subseteq R_{F} \bar{I} \subseteq R_{F}$ with each a dense submodule of its successor. Hence $\sum_{i=1}^{t} R_{F} \bar{a}_{i}$ is an injective $R$-module which is dense in $R_{F}$. By (1.2), $\sum_{i=1}^{t} R_{F} \bar{a}_{i}=R_{F} \bar{I}=R_{F}$. (Thus $F$ is a perfect topology, in the sense of [8, p. 231].)

It follows that every $R_{F}$-module is torsion-free (for if $x$ is an element of an $R_{F}$-module and $I x=0$ with $I \in F$, then $\left.R_{F} x=R_{F} I_{x}=0\right)$ ). In particular, every left ideal of $R_{F}$ is closed, hence is torsion-free and $F$-injective. By hypothesis, then, every left ideal of $R_{F}$ is $R$-injective. Since $R$-injective implies $R_{F}$-injective for torsion-free $R_{F}$-modules, this completes the proof. $/ /$

We remark that, in the preceding theorem, we could replace the requirement that $F$ is cofinally finite by the stronger hypothesis that $F$ 
be perfect (that is, $R_{F} I=R_{F}$ for all $I \in F$ ). This was noted in the course of proving the implication (3) $\Rightarrow(1)$.

It is now our intention to examine for a moment the hypotheses of Theorem 2.1 in order to determine their relationship with other familiar conditions. For instance, $F$ being cofinally finite is a weaker condition than the ascending chain condition on closed left ideals. Their precise correlation is now given. We let $\mathcal{C}_{F}(R)$ denote the lattice of closed left ideals of $R$.

PROPOSITION 2.2. The following conditions are equivalent:

(i) $R$ satisfies the ascending chain condition on closed left ideals;

(ii) $F$ and $C_{F}(R)$ are cofinally finite;

(iii) the set of alz left ideals of $R$ is cofinally finite.

Proof. The equivalence of $(i)$ and $(i i i)$ is noted as part of Proposition XIII.2.4 in [8], and we have proved that $(i) \Rightarrow$ (iii) in $(2) \Rightarrow(3)$ of the preceding theorem. For the sake of completeness, then, we will demonstrate that $(i i) \Rightarrow(i)$.

Let $J_{1} \subseteq J_{2} \subseteq \ldots$ be a sequence of closed left ideals. Set

$J=\bigcup_{i=1}^{\infty} J_{i}$; we first show that $J$ is closed.

Suppose that $J$ is dense in $I \subseteq R$. Let $a \in I$ be arbitrary. Then $(J: a) \in F$, so by hypothesis there exists $I_{0}=R a_{1}+\ldots+R a_{t}$ dense in $(J: a)$. For each $k=1, \ldots, t, a_{k} a \in J$, so there exists an integer $n$ with $I_{0} a \subseteq J_{n}$. Since $I_{0} \in F$ and $J_{n}$ is closed, $a \in J_{n}$. Thus $J=I$ and $J$ is closed.

ivext, we know by hypothesis that there exists $R b_{1}+\ldots+R b_{s}$ dense in $J$. But then there is an integer $m$ with $R b_{1}+\ldots+R b_{s} \subseteq J_{m}$. This implies that $J_{m}$ is dense in $J$. Since $J_{m}$ is closed, $J_{m}=J$, and the sequence is finite. //

As we have seen in (1.2), a dense submodule of a torsion-free module 
is an essential submodule. The converse statement (that is that essential submodules of torsion-free modules be dense) can be seen to be equivalent to torsion-free F-injectives being injective. The proof is a simple variant of the one given for Proposition 2.4 in [4], and will therefore not be presented here.

(2.3). Torsion-free F-injective modules are injective if and only if essential submodules of torsion-free modules are dense.

\section{3.}

Throughout this section we let $G$ denote the Goldie topology, $G=\left\{\left.{ }_{R} I\right|_{R} ^{I \subseteq} \subseteq_{R}^{J}\right.$ where $J$ and $(I: a)$ are essential left ideals of $R$ for every $a \in J\}$;

that is, $G$ is the smallest Gabriel topology which contains the set of essential left ideals of $R$ (see [8, p. 148]). G-injective modules are of course injective, and so Theorem 2.1 specializes as follows.

THEOREM 3.1. FOr $F$ any Gabriel topology containing $G$, the following conditions are equivalent:

(I) $R_{F}$ is semisimple artinian;

(2) $C_{F}(R)$ satisfies the ascending chain condition;

(3) F is cofinally finite;

(4) there is no infinite independent family of F-torsion-free left ideals of $R$.

Since $F \supseteq G$ is equivalent to $F$-injectives being injective, $R_{F}$ is semisimple artinian with $F \supseteq G$ if and only if $F$ is cofinally finite and F-injectives are injective.

(1), (2), and (3) are equivalent by Theorem 2.1. The proof that $(3) \Rightarrow(4) \Rightarrow(2)$ involves a rather standard argument which appears also in Proposition XIII.3.1 of [8], where additional equivalent conditions are listed. We will therefore omit the proof.

LEMMA 3.2. Assume that torsion-free F-injective modules are injective. Then $\bar{F}=$ \{essential left ideals of $\bar{R}\}$, the left singular 
ideal of $\bar{R}=R / T(R)$ is zero, and $R_{F}$ is the maximal left quotient ring of $\bar{R}$.

Proof. Let $\bar{I}$ be an essential left ideal of $\bar{R}$. By (2.3) and Lemma 1.5 (i), $\bar{I} \in \bar{F}$. Conversely, $\bar{I} \in \bar{F}$ implies that $\bar{I}$ is essential in $\bar{R}$ by (1.2). Since $T_{\bar{F}}(\bar{R})=\overline{0}$ by Lemma 1.5 (iii), the right annihilator of $\bar{I}$ in $\bar{R}$ equals zero. This proves that the left singular ideal of $\bar{R}$ is zero.

As is well-known, the maximal left quotient ring of a nonsingular ring is just the injective hull [8, p. 149]. Now because of the hypothesis and Lemma 1.5 (iv), $R_{F}$ must be the maximal left quotient ring of $\bar{R}_{R}$. //

Combining these results we obtain the following consequence.

COROLLARY 3.3. If $R$ is a finite dimensional ring and $F \supseteq G$, then $R_{F}$ is semisimple artinian and is the maximal left quotient ring of $R / T_{F}(R)$.

\section{4.}

Another important special case of the main theorem occurs when $F$ is cofinitely principal; that is, when each left ideal in $F$ contains a principal left ideal in $F$.

Recall that when $S$ is a multiplicatively closed subset of a ring $R$, a classical left quotient ring of $R$ with respect to $S$ is defined to be a ring $R_{S}$ together with a ring homomorphism $\varphi: R \rightarrow R_{S}$ such that

(i) $\varphi(s)$ is a unit in $R_{S}$ for each $s \in S$;

(ii) every element of $R_{S}$ has the form $\varphi(s)^{-1} \varphi(a)$ with $s \in S, a \in R ;$ and

(iii) $\varphi(a)=0$ if and only if $s a=0$ for some $s \in S$.

It can be established [8, p. 51] that the classical left quotient ring of $R$ with respect to $S$ exists if and only if $S$ satisfies:

(a) if $s \in S$ and $a \in R$, then there exists $t \in S$ and $b \in R$ with $t a=b s ;$ and 
(b) if $a s=0$ with $a \in R, s \in S$, then $t a=0$ for some $t \in S$.

Such a multiplicatively closed set $S$ will be called a left denominator set. For the usual reasons, when $R_{S}$ exists, it is unique up to isomorphism over $R$; furthermore, $F=\left\{_{R} I \mid I \cap S \neq \emptyset\right\}$ is then a cofinitely finite Gabriel topology and $R_{F}=R_{S} \quad[8$, p. 238].

PROPOSITION 4.1. Assume that $F$ is cofinitely principal and that F-torsion-free $F$-injectives are injective. Then $R_{F}$ is semisimple artinian and is the classical left quotient ring of $R$ with respect to $S=\{s \in R \mid R s \in F\}$.

Proof. It is well-known and easy to prove that for a cofinitely principal Gabriel topology $F, S=\{s \in R \mid R s \in F\}$ is a multiplicatively closed subset of $R$ satisfying (a) [8, p. 237]. To see that (b) holds, suppose that $a s=0$ with $a \in R, s \in S$. From $\S 2$, we know that $F$ is a perfect topology, so $R_{F} \bar{s}=R_{F}$ where $\bar{s}=s+T(R) \in R / T(R)$. Since $R_{F}$ is artinian, $\bar{s}$ is a unit of $R_{F}$. Now as $=0$ implies that $\bar{a} \bar{s}=\overline{0}$, whence $a \in T_{F}(R)$. It follows that there exists $t \in S$ with $t a=0$. Thus we know that $R$ has a classical left quotient ring with respect to $s$. //

The preceding proposition extends Theorem 1.7 of [7].

A result due to Levy states that if a ring $R$ has a classical left quotient ring $Q$, then $Q$ is semisimple artinian if and only if torsionfree divisible $R$-modules are injective [5, Theorem 3.3]. In Theorem 4.3 we provide a generalization of this to classical left quotient rings with respect to left denominator sets.

Given any $S \subseteq R$ and a module $R^{M}$, we call $M S$-torsion-free if whenever $s m=0$ with $s \in S$ and $m \in M$, then $m=0$; and $M$ is called S-divisible if $s M=M$ for each $s \in S$.

LEMMA 4.2. If $S$ is a left denominator set and $I$ is a left ideal of $R$ then there is a natural isomorphism $R_{S} \otimes_{R} \varphi(I) \cong R_{S} \varphi(I)$, where $\varphi$ denotes the canonical homomorphism of $R$ into $R_{S}$. 
Proof. The isomorphisms $R_{S} \otimes_{R} \varphi(I) \underset{v}{\stackrel{\mu}{\rightleftarrows}} R_{S} \varphi(I)$ are defined by $\mu\left(\sum_{i=1}^{n} x_{i} \otimes \varphi\left(a_{i}\right)\right)=\sum_{i=1}^{n} x_{i} \varphi\left(a_{i}\right)$ and $\nu\left(\sum_{i=1}^{n} x_{i} \varphi\left(a_{i}\right)\right)=\sum_{i=1}^{n} x_{i} \otimes \varphi\left(a_{i}\right)$, where $x_{i} \in R_{S}, a_{i} \in I . \mu$ is well-defined by the universal mapping property of tensor products, and the proof is completed by showing that $v$ is well-defined.

To see this, let $\sum_{i=1}^{n} x_{i} \varphi\left(a_{i}\right)=0$ with $x_{i} \in R_{S}, a_{i} \in I$. We may choose a common denominator $s \in S$ and $b_{1}, \ldots, b_{n} \in R$ so that each $x_{i}=\varphi(s)^{-1} \varphi\left(b_{i}\right)$. Then $\sum_{i=1}^{n} \varphi\left(b_{i}\right) \varphi\left(a_{i}\right)=0$, and so $\varphi(s)\left(\sum_{i=1}^{n} x_{i} \otimes \varphi\left(a_{i}\right)\right)=\sum_{i=1}^{n} \varphi\left(b_{i}\right) \otimes \varphi\left(a_{i}\right)$ $=\sum_{i=1}^{n} \varphi(1) \otimes \varphi\left(b_{i}\right) \varphi\left(a_{i}\right)=\varphi(1) \otimes \sum_{i=1}^{n} \varphi\left(b_{i}\right) \varphi\left(a_{i}\right)=0$. Since $\varphi(s)$ is a unit, $\sum_{i=1}^{n} x_{i} \otimes \varphi\left(a_{i}\right)=0$, and $\nu$ is well-defined. //

THEOREM 4.3. Let $S$ be a left denominator set in $R$. Then $R_{S}$ is semisimple artinian if and only if S-torsion-free S-divisible R-modules are injective.

Proof. Set $F=\left\{t_{R} I I \cap S \neq \emptyset\right\}$. It is straightforward to check that a module is F-torsion-free if and only if it is $S$-torsion-free. We begin by showing that the $F$-torsion-free $F$-injectives are precisely the $S$-torsion-free $S$-divisibles.

Suppose that $M$ is $F$-torsion-free and F-injective and let $s \in S$, $m \in M$ be given. Define $f \in \operatorname{hom}_{R}(R s, M)$ by $f(a s)=a m, a \in R$. $f$ is well-defined; for $a s=0$ implies that there exists $t \in S$ with $t a=0$, and so $\operatorname{tam}=0$, whence $a m=0$, since $M$ is F-torsion-free. Since $M$ is F-injective there exists $g \in \operatorname{hom}_{R}(R, M)$ with $\left.g\right|_{R s}=f$. Now $m=f(s)=g(s)=s g(1)$, and this proves that $M$ is $S$-divisible. 
Conversely, assume that $M$ is $S$-torsion-free and $S$-divisible, and let $f \in \operatorname{hom}_{R}(I, M)$ be given with $I \in F$. Choose $s \in I \cap S ; f(s)=m$ for some $m \in M$. Since $M$ is $S$-divisible, we may choose $n \in M$ with $m=s n$. Now define $g \in \operatorname{hom}_{R}(R, M)$ by $g(r)=m$. We claim that $\left.g\right|_{I}=f$. For given $a \in I$, there exists $t \in S, b \in R$ with ta $=b s$. So

$$
\begin{array}{r}
t(f(a)-g(a))=f(t a)-g(t a)=f(b s)-g(b s) \\
\quad=b f(s)-b g(s)=b m-b s n=0 .
\end{array}
$$

Since $M$ is S-torsion-free, $f(a)=g(a)$, and so $M$ is F-injective.

Now assume that $S$-torsion-free $S$-divisible modules are injective. By Proposition 4.1, $R_{S}=R_{F}$ is semisimple artinian.

Conversely, assume that $R_{S}$ is semisimple artinian, and let $R^{M}$ be $S$-torsion-free and $S$-divisible. Then $M$ is an $R_{S}$-module. For given $m \in M$ and $x=\varphi(s)^{-1} \varphi(a) \in R_{S}$ with $s \in S, a \in R$, there exists a unique element $n \in M$ with $a m=s n$; and defining $x \cdot m=n$ determines the $R_{S}$-module structure of $M$, as can be readily verified.

Now let $f \in \operatorname{hom}_{R}(I, M)$ be given, with $I$ a left ideal of $R$. Then $J=\operatorname{ker} \varphi \cap I=\{a \in I \mid s a=0$ for some $s \in S\}$ is a left ideal, and $f(J)=0$ because $M$ is $S$-torsion-free. Hence $f$ induces $f^{\prime} \in \operatorname{hom}_{R}(\varphi(I), M)$ with $f^{\prime} \circ \varphi=f$. By Lemma 4.2, $R_{S} \otimes_{R} \varphi(I) \cong R_{S} \varphi(I)$, and therefore $f^{\prime}$ can be extended to $g^{\prime} \in \operatorname{hom}_{R}\left(R S^{\varphi}(I), M\right)$. Since $R_{S^{\varphi}}(I)$ is a left ideal of the semisimple artinian ring $R_{S}, g^{\prime}$ can be extended to $g \in$ hom $_{R}\left(R_{S}, M\right)$. But then $g \circ \varphi \in \operatorname{hom}_{R}(R, M)$ extends $f$. So $R^{M}$ is injective, and the proof is complete. //

5.

The next objective is to examine ideals of $R_{F}$ in the case when torsion-free $F$-injectives are injective. Some observations can be made in 
a more general setting.

Assume that $F$ and $G$ are Gabriel topologies with $F \subseteq G$. Then $T_{F}(R) \subseteq T_{G}(R)$; and we let $p$ denote the canonical homomorphism from $R / T_{F}(R)$ onto $R / T_{G}(R)$. Consider the diagram

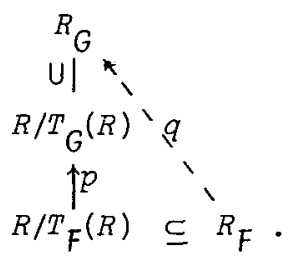

Since $R_{G}$ is $F$-torsion-free and $F$-injective there exists a unique $R$-homomorphism $q: R_{F} \rightarrow R_{G}$ extending $p$.

We claim that $q$ is in fact a ring homomorphism. To see this, let $\bar{r}$ denote the coset of $r \in R$ in $R / T_{F}(R)$, and observe that the $R$-module structures of $R_{F}$ and $R_{G}$ are defined by $r \cdot \alpha=\bar{r} \alpha, \quad r \cdot \gamma=p(\bar{r}) \gamma$ for any $r \in R, \quad \alpha \in R_{F}, \gamma \in R_{G}$. Now let $\alpha, \beta$ be arbitrary elements of $R_{F}$ and set $I=\left(R / T_{F}(R): \alpha\right) \in F$. Then for any $r \in I$, $r(q(\alpha \beta)-q(\alpha) q(\beta))=q(r \cdot \alpha \beta)-q(r \cdot \alpha) q(\beta)$ $=q(r \alpha \cdot \beta)-p(r \alpha) q(\beta)=p(r \alpha) q(\beta)-p(r \alpha) q(\beta)=0$

since $r \alpha \in R / T_{F}(R)$ and $q$ extends $p$. Since $r \in I$ was arbitrary and $R_{G}$ is F-torsion-free, it follows that $q(\alpha \beta)=q(\alpha) q(\beta)$. We summarize this.

(5.1). If $F \subseteq G$ are Gabriel topologies, then there is a unique $R$-algebra homomorphism $q: R_{F} \rightarrow R_{G}$ which extends the cononical epimorphism $p: R / T_{F}(R) \rightarrow R / T_{G}(R) \cdot$ (See [8, p. 210, Exercise 1].)

PROPOSITION 5.2. Suppose that F-injective ideals of $R_{F}$ are $R$-injective, and let $G$ be a cabriel topology with $F \subseteq G$. Then $R_{G}$ is isomorpinic to a direct summand of $R_{F}$ under a splitting of the homomorphism $q$ of (5.1). 
Proof. Let $q: R_{F} \rightarrow R_{G}$ be the algebra homomorphism described by (5.1). Since $R_{G}$ is F-torsion-free, kernel $q$ is F-injective, and so by hypothesis $R_{F}$ and kernel $q$ are injective $R$-modules. Hence $R_{F} \cong$ kernel $q \oplus q\left(R_{F}\right)$, from which we have that $q\left(R_{F}\right)$ is injective. But $R / T_{G}(R) \subseteq q\left(R_{F}\right)$, so necessarily $q\left(R_{F}\right)=R_{G}$. //

A converse is true as well.

PROPOSITION 5.3. If $\underline{\underline{A}}$ is an ideal direct summand of $R_{F}$ which is injective as a left R-module, then $\mathrm{A}$ is a quotient ring of $R$ with respect to some Gabriel topology $G \supseteq F$.

Proof. Choose an ideal $\underline{\underline{B}}$ with $R_{F}=\underline{\underline{A}} \oplus \underline{\underline{B}}$. Set $T=\left\{R_{R}^{M} \mid \operatorname{hom}_{R}(M, \underline{A})=0\right\} ; T$ is a hereditary torsion class containing the F-torsion modules because $A$ is an injective F-torsion-free $R$-module. Let $G$ be the Gabriel topology of left ideals associated to $T$; then $G \supseteq F$ and $T_{G}(R) \supseteq T_{F}(R)$. We will show that $R_{G} \cong \underline{\underline{A}}$.

Let $q: R_{F} \rightarrow R_{G}$ be the ring homomorphism of (4.1). Since $\operatorname{hom}_{R_{F}}(\underline{\underline{B}}, \underline{\underline{A}})=0, \operatorname{hom}_{R}(\underline{\underline{B}}, \underline{\underline{A}})=0$. So $\underline{\underline{B}} \in T$, and hence $\underline{\underline{B}} \subseteq$ kernel $q$. If $\underline{\underline{A}} \cap$ ker $q \neq 0$, then $A=\underline{\underline{A}} \cap \operatorname{ker} q \cap R / T_{F}(R) \neq 0$, and consequently $A p=A_{q}=0$. Hence $A \subseteq$ kernel $p=T_{G}(R) / T_{F}(R) \in T$. But then $\operatorname{hom}_{R}(A, \underline{\underline{A}})=0$, which forces $A=0$, a contradiction. Thus it must be the case that $\underline{\underline{A}} \cap$ ker $q=0$, and so $\left.q\right|_{\underline{A}}$ is a monomorphism. Next $q\left(R_{F}\right)=q(\underline{\underline{A}})$ is an injective $R$-submodule of $R_{G}$ which contains $R / T_{G}(R)$, and we conclude from this that $q(\underline{A})=R_{G}$. So $\cong \cong R_{G}$ under the homomorphism $q$. //

An ideal $T$ of a ring $R$ is a torsion ideal if there exists a proper Gabriel topology $G$ with ${ }_{G}(R)=T$. We can now apply the information above to learn when a semisimple artinian ring of quotients is simple.

THEOREM 5.4. Assume that $R_{F}$ is semisimple artinian. Then the 
following conditions are equivalent:

(i) $R_{F}$ is simple;

(ii) $R_{F}=R_{G}$ for every proper Gabriel topology $G \supseteq F$;

(iii) $T_{F}(R)$ is a maximal torsion ideal.

Proof. $(i) \Rightarrow(i i i)$. Suppose that $R_{F}$ is simple, and say $T_{F}(R) \varsubsetneqq T_{G}(R)$ for some Gabriel topology $G$. Then $\bar{T}=\overline{T_{G}(R)}$ is a nonzero G-torsion submodule of $\bar{R}=R / T_{F}(R)$. Since ${ }^{R} F$ is simple, ${ }^{R} \bar{F} R_{F}=R_{F}$. Write $I=\sum_{i=1}^{t} \alpha_{i} \bar{t}_{i} \beta_{i}$ with $\alpha_{i}, \beta_{i} \in R_{F}, \bar{t}_{i} \in \bar{T}$. Set $J=\bigcap_{i=1}^{t}\left(\bar{R}: \alpha_{i}\right) \in F$. Then for any $a \in J$,

$$
\bar{a}=\bar{a} \sum_{i=1}^{t} \alpha_{i} \bar{t}_{i} \beta_{i}=\sum_{i=1}^{t}\left(\bar{a} \alpha_{i}\right) \bar{t}_{i} \beta_{i} \subseteq \bar{R} \cap \bar{T} R_{F} \subseteq T_{G}(\bar{R})
$$

It follows that $\bar{J} \subseteq T_{G}(\bar{R})$, and hence by $(1.2),{ }^{T}{ }_{G}(\bar{R})$ is an essential left ideal of $\bar{R}$. But $T_{G}(\bar{R}) \subseteq T_{G}\left(R_{F}\right)$, so $T_{G}\left(R_{F}\right)$ must be an essential ideal of $R_{F}$. Hence $T_{G}\left(R_{F}\right)=R_{F}$. By considering again the identity element of $R_{F}$, this forces $T_{G}(R) \in G$, which is impossible unless $0 \in G$.

(iii) $\Rightarrow$ (ii). This is clear from Proposition 5.2.

$(i i) \Rightarrow(i)$. If $R_{F}$ is not simple, let $\underline{\underline{A}}$ be any proper ideal of $R_{F}$. By Proposition 5.3, there exists a Gabriel topology $G \supseteq F$ with $R_{G}=\underline{\underline{A}} \varsubsetneqq R_{F}$

We conclude this section with the following result, which is actually a consequence of the material in $\$ 2$.

THEOREM 5.5. $R_{F}$ is a division ring if and only if $T_{F}(R)$ is the only proper closed left ideal of $R$.

Proof. Suppose that $R_{F}$ is a division ring, and that $I$ is a closed 
left ideal of $R$. If $I \neq T(R)$, then $R_{F} \bar{I}$ is a nonzero left ideal of $R_{F}$, so $R_{F} \bar{I}=R_{F}$. But then $I$ is clearly dense in $R$, so, necessarily, $I=R$.

Conversely, assume that $T(R)$ is the only closed left ideal of $R$, other than $R$. We first show that a torsion-free F-injective module $M$ is injective. For let $f \in \operatorname{hom}_{R}(I, M)$ with $I$ a left ideal of $R$. By our hypothesis either $I \subseteq T(R)$, in which case $f=0$, or else $I \in F$. In any event, $f$ can be extended to an element of $\operatorname{hom}_{R}(R, M)$, and so $R^{M}$ is injective.

By Theorem 2.1 (2), $R_{F}$ is semisimple artinian; and from the discussion immediately following that theorem, we know that $F$ is perfect. Let $K$ now be any left ideal of $R_{F}$. Since $F$ is perfect, $K$ is closed in $R_{F}$. It follows that $I=\psi^{-1}(K \cap \bar{R})$ is closed in $R$, where $\psi: R \rightarrow R_{F}$ is the canonical homomorphism. By hypothesis, $I=T(R)$ or $I=R$, and from this we can conclude that $K=0$ or $K=R_{F}$. Thus $R_{F}$ is a division ring. //

6.

In this final section we treat some related facts, which extend results known for classical rings of quotients. For instance, a ring with a simple (respectively, semisimple) artinian classical ring of quotients is prime (respectively, semiprime). More generally, we have the following:

PROPOSITION 6.7. Suppose that $R_{F}$ is a simple ring respectively, a finite direct sum of simple rings), and that every left ideal in $\bar{F}$ is a faithful $\bar{R}$-module. Then $T_{F}(R)$ is a prime (respectively, semiprime) ideal of $R$.

Proof. First assume that $R_{F}$ is simple, and let $A B \subseteq T_{F}(R)$ with $A$ and $B$ left ideals of $R$. and $B \notin T_{F}(R)$. Then $\bar{B} \neq \overline{0}$ in $\bar{R}=R / T_{F}(R)$, so $R_{F} \bar{B}_{F}=R_{F}$ by the hypothesis on $R_{F}$. Write $1=\sum_{i=1}^{t} p_{i} \bar{b}_{i} q_{i}$ with 
$p_{i}, q_{i} \in R_{F}, \quad b_{i} \in B$. Let $I=\bigcap_{i=1}^{t}\left(\sqrt[R]{ }: p_{i}\right) \in F$. Now $\bar{I}=\bar{I} I \subseteq \sum_{i=1}^{t}\left(I p_{i}\right) \bar{b}_{i} q_{i} \subseteq \bar{R} \bar{B} R_{F} \subseteq \bar{B} R_{F}$. Hence $\bar{A} \bar{I} \subseteq \bar{A} \bar{B} R_{F}=\overline{0}$. Since $\bar{R}$ is faithful, we conclude that $\vec{A}=\overline{0}$, proving that $T_{F}(R)$ is a prime ideal.

For the semisimple case, let $B$ be a left ideal of $R$ with $B^{n} \subseteq T_{F}(R), n \geq 1$. We may write $R_{F} \bar{B} R_{F}=R_{F} e$ with e a central idempotent of $R$. As above, write $e=\sum_{i=1}^{t} p_{i} \bar{b}_{i} q_{i}$ and let $I=\bigcap_{i=1}^{t}\left(\bar{R}: p_{i}\right) \in F$. Then $e \bar{I}=\bar{I}_{e} \subseteq \bar{B}_{F}$, so that $\bar{B}^{n-1} e \bar{I} \subseteq \bar{B}^{n}=\overline{0}$. Since $\bar{I}$ is faithful, $\vec{B}^{n-1} e=\overline{0}$. But $e$ is an identity element on $\bar{B}$, so $\vec{B}^{n-1}=\overline{0}$. Continuing in this manner, we eventually learn that $\bar{B}=\overline{0}$, and this proves that $T_{F}(R)$ is a semiprime ideal. //

COROLLARY 6.2. If $S$ is a left denominator set such that $R_{S}$ is a simple ring (respectively, a direct sum of simple rings), then $T(R)=\{a \in R \mid s a=0$ for some $s \in S\}$ is a prime (respectively, semiprime) ideal.

Let us call a topology $F$ hereditary if every left ideal in $F$ is projective. Cofinally finite hereditary topologies (which are not necessarily Gabriel topologies) often consist of finitely generated left ideals, as we now see.

PROPOSITION 6.3. Assume that $T_{F}(R)=0$ and that $F$ is a cofinally finite hereditary topology. Then every $I \in F$ is finitely generated.

Proof. Let $I \in F$ be given. Choose $J=\sum_{i=1}^{t} R x_{i} \subseteq I$ with $J \in F$. By hypothesis $I$ is projective, so we may choose $f_{\alpha} \in \operatorname{hom}_{R}(I, R)$, $y_{\alpha} \in I$ with $y=\sum_{\alpha \in A}\left(y f_{\alpha}\right) y_{\alpha}$ for each $y \in I$. Since $J$ is finitely 
generated, $\left.f_{\alpha}\right|_{d}=0$ for all but finitely many $\alpha \in A$. For simplicity, let us assume that $\left.f_{\alpha}\right|_{J}=0$ for $\alpha \notin\{1,2, \ldots, k\}$. Now for each $\alpha \vDash\{1,2, \ldots, k\}, f_{\alpha}$ induces $f_{\alpha}^{\prime}: I / J \rightarrow R$ and $(I / J) f_{\alpha}^{\prime}$ is F-torsion. Since $T_{F}(R)=0, f_{\alpha}^{\prime}=0$; that is, $f_{\alpha}=0$ for each $\alpha \vDash\{1,2, \ldots, k\}$, and it follows that $y_{1}, y_{2}, \ldots, y_{k}$ generate $I . / /$ COROLLARY 6.4 [8, p. 260]. A finite dimensional hereditary ring is noetherian.

Proof. A hereditary ring is nonsingular. So

$F=$ \{essential left ideals $\}$ forms a Gabriel topology satisfying the previous proposition. It follows that every left ideal, being a summand of an essential left ideal, is finitely generated.

\section{References}

[1] В.П. Елизаров [V.P. Elizarov], "Артнновы нольца частных" [Artinián rings of quotients], Uspehi Mat. Nauk 30 (1975), no. 2 (182), $211-212$.

[2] Jonathan S. Golan, Localization of noncommutative rings (Marcel Dekker, New York, 1975).

[3] Garry Helzer, "On divisibility and injectivity", Canad. J. Math. 18 (1966), 901-919.

[4] Joachim Lambek, "Localization and completion", J. Pure Appl. Algebra 2 (1972), 343-370.

[5] Lawrence Levy, "Torsion-free and divisible modules over non-integraldomains", Canad. J. Math. 15 (1963), 132-151.

[6] S. Page, "Properties of quotient rings", Canad. J. Math. 24 (1972), $1122-1128$.

[7] Francis L. Sandomierski, "Semisimple maximal quotient rings", Trans. Amer. Math. Soc. 128 (1967), 112-120. 
[8] Bo Stenström, Rings of quotients. An introduction to methods of ring theory (Die Grundlehren der mathematischen Wissenschaften, 217. Springer-Verlag, Berlin, Heidelberg, New York, 1975).

Department of Mathematics,

University of Cal ifornia, Santa Barbara,

Santa Barbara,

Cal i fornia,

USA. 